\title{
TEORES DE ROTENONA EM CLONES DE TIMBÓ (Derris spp. FABACEAE) DE DIFERENTES REGIÕES DA AMAZÔNIA E OS SEUS EFEITOS NA EMERGÊNCIA DE IMAGOS EM Musca domestica $\mathrm{L}$.
}

\author{
José Paulo Chaves da COSTA ${ }^{1}$, Sérgio de Mello ALVES', Muracy BÉLO ${ }^{2}$
}

RESUMO - A análise dos teores de rotenona, em clones de três espécies de timbó, permitiram a classificação destas plantas de acordo com as suas eficiencias no controle de larvas de $M u s c a$ domestica. Os resultados evidenciaram correlações significativas entre os teores de rotenona apresentados pelos clones de Derris urucu e de Derris nicou, com relação a capacidade de controle das larvas. As plantas com os maiores teores de rotenona foram as mais eficientes. O conteúdo de rotenona, os efeitos dos clones das espécies de Derris nas moscas, além dos locais de origem das plantas, mostraram que deve ter ocorrido entre estes timbós, a existência de isolamento populacional, durante a época do pleistoceno na Amazônia. Em Derris sp., que apresentou menor teor de rotenona, sendo ineficiente no controle das larvas, estas diferenças não foram assinaladas.

Palavras chaves: Timbó, rotenona, Musca domestica, Amazônia, refúgios florestais.

Rotenone Contents in Timbó Clones (Derris spp.: Fabaceae) from Different Amazonian Regions and their Effects on the Emergence of Musca domestica L. Imagoes.

ABSTRACT - The rotenone contents analysis in clones of three timbó species permited the classification of these plants based on their efficiency to control Musca domestica larvae. The results showed significant correlations between the rotenone contents in the Derris urucu and D. nicou clones with the capacity to control the larvae. The plants with high rotenone contents were more effective. The rotenone contents, the effect of Derris species clones on the flies and the localization of the plants origin, showed that among the plants could have happened a populational isolation in the Amazonia during the pleistocene epoch. In Derris sp., that presented lower rotenone contents and was ineffective to control of the larvae, these differences were not observed.

Key-words: Timbó, rotenone, control, Musca domestica, Amazonia, florest refugia.

\section{INTRODUÇÃO}

Os indigenas da América do Sul, particularmente da Amazônia, fazem suas pescas com o uso de timbó, principalmente quando maior quantidade de alimento seja necessário (Corbett, 1940). Estas plantas são cipós trepadores que atingem a copa das árvores e que, de acordo com a revisão feita por Francis Macbride, foram retiradas do gênero Lonchocarpus e colocadas no gênero
Derris, dentro da familia das Leguminosas (Lima, 1987).

Há muitas espécies de timbó, mas as de uso mais generalizado são D. итиси e D. nicou, que segundo Lima (1987), são portadoras nas raizes da substância rotenona, de onde deriva a importância dessas plantas.

Antes de 1946, a rotenona era utilizada como inseticida nas lavouras contra insetos (larvas de borboletas, coccideos, cochonilhas e pulgões) e

${ }^{1}$ EMBRAPA - Amazônia Oriental, Caixa Postal 48, 66017-970, Belém, PA.

${ }^{2}$ FCAVJ-UNESP, 14870-000, Jaboticabal, SP. 
ectoparasitas de animais. Costa et al. (1986) relata o sucesso de D. urucu no controle do piolho Haematopinus tuberculatus, dos búfalos. A despeito da rotenona ser de um modo geral, inócua para animais de sangue quente (Lima, 1987), ela pode ser absorvida pela pele de mamíferos, porém, relativamente inofensiva quando utilizada adequadamente (Link, 1965). Vianna et al. (1976) verificaram que extratos alcóolicos com rotenona de Derris negresis aplicados por via cutânea, foram mais eficientes que os extratos aquosos e produziram sinais evidentes de intoxicação e morte após sete dias em camundongos. Os efeitos tóxicos da rotenona foram manifestados por conjuntivite, faringite, dermatite, irritação gastrointestinal e pulmonar, náuseas e vômitos.

As exportações de timbó iniciaram-se em 1939. O produto exportável era o pó das raízes e havia vários moinhos em operação em Manaus (AM) e Belém (PA). Com o advento do DDT e dos inseticidas sintéticos, o comércio do pó das raizes de timbó entrou em colapso, juntamente com a atividade de pesquisa (Pires, 1978).

O objetivo deste trabalho é de analisar o teor de rotenona no pó das raízes de clones em três espécies de Derris de diferentes regiões da Amazônia e os seus efeitos em Musca domestica. Plantas de determinadas regiões foram intencionalmente selecionadas, com o propósito de detectar possiveis existências de isolamento, que poderia ter ocorrido anteriormente a época atual na Amazônia, pois os timbós são fortes indicadores de florestas úmidas.

\section{MATERIAL E MÉTODOS}

No período de janeiro/84 a dezembro/88, a Empresa Brasileira de Pesquisa Agropecuária (Embrapa), através do Centro de Pesquisa Agroflorestal da Amazônia Oriental, realizou diversas expedições para coletas de germoplasma de plantas amazônicas, estando os timbós relacionados entre as espécies destinadas ao trabalho de coletas (Lima \& Costa, 1991).

A amostragem foi feita através de ramos (estacas) destacados de plantas matrizes. Aqueles provenientes de uma mesma planta receberam sempre a mesma numeração. Em seguida, todo material foi remetido à Embrapa para plantio, fazendo parte hoje do Banco Ativo de Germoplasma da instituição. As raizes empregadas no trabalho, foram obtidas das plantas cultivadas em Belém (PA) em área com o mesmo tipo de solo, sendo selecionadas as amostras provenientes originalmente de regiões consideradas "refúgios florestais" ou mais próximos a eles, como foi descrito por Haffer (1967 e 1969) para a Amazônia, durante o período do pleistoceno (Fig. 1).

Neste trabalho foram utilizadas as espécies: D, urucu, D. nicou e Derris sp., conhecidos nas regiões de coletas, pela denominação vulgar de timbó vermelho, branco e timbó amarelo ou melancia, respectivamente.

Os testes foram realizados com 


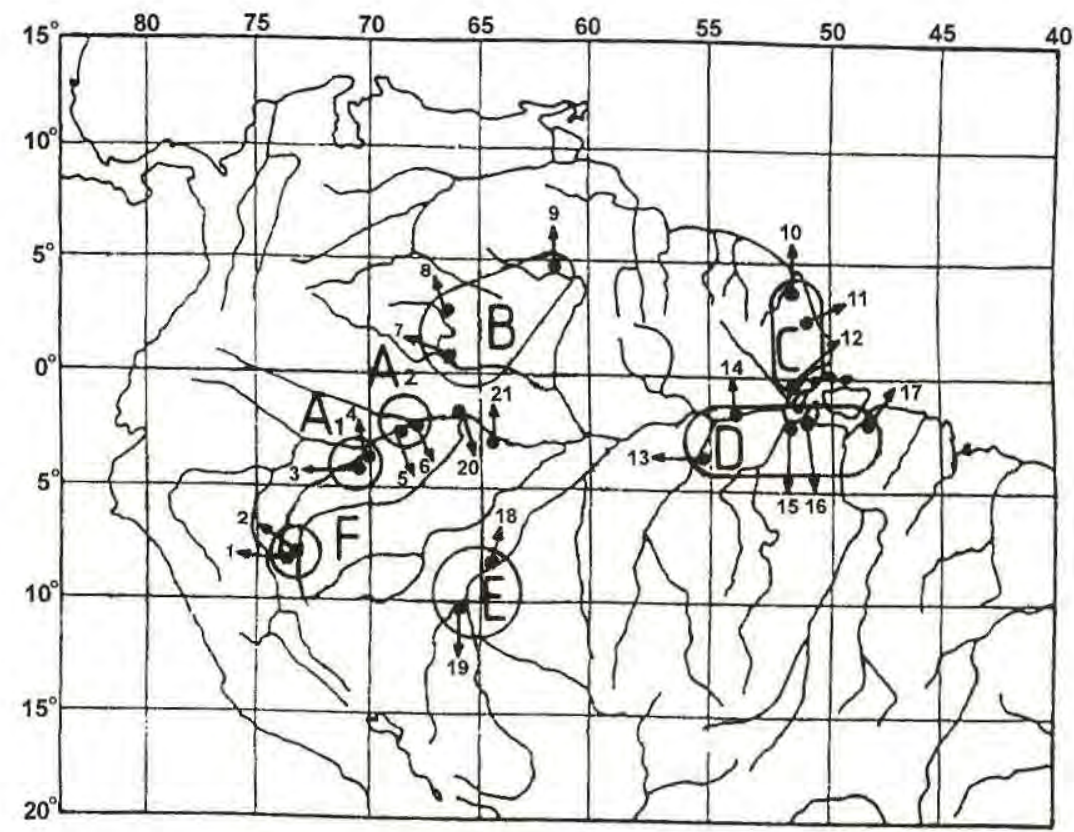

Legenda:

1: Mâncio Lima; 2: Cruzeiro do Sul: 3: Benjamim Constant; 4: Tabatinga; 5 : Såo Pauulo de Olivença; 6 : Santo Antônio do Içá; 7: São Gabriel da Cachoeira: 8: Santa Rosa (Venezuela): 9: Marco BV-8; 10: Oiapoque; 11 : Calçoene; 12: Mazagāo; 13: Aveiro; 14: Monte Alegre; 15: Porto de Moz; 16: Gurupá; 17: Belém; 18: Humaitá; 19: Costa Marques; 20: Fonte Boa; e, 21:Tefé

Figura 1. Mapa da parte norte da América do Sul, mostrando a bacia amazônica, com as regiōes $\left(A_{1}, A_{2}, B, C, D, E\right.$ e F) e os municípios (Legenda), onde as coletas de timbós foram realizadas.

o pó das raizes, que foram seccionadas a $30 \mathrm{~cm}$ da cepa. Depois de limpas foram cortadas em pedaços de $25 \mathrm{~cm}$, amarradas em amostras e etiquetadas com a identificação do número do clone. Posteriormente, foram submetidas a processo de secagem, utilizando-se estufa com ventilação forçada, em temperatura constante de 40 $\pm 20 \mathrm{C}$, sendo as amostras pesadas diariamente até a estabilização do peso. Em seguida, foram trituradas separadamente, em um micro-moinho tipo Willey com o uso de uma peneira de 40 mesh, para a formação do pó, sendo o produto de cada clone, armazenado em recipientes de plástico leitoso, não transparente e conservado em ambiente escuro com circulação de ar.

As determinações dos teores de rotenona foram realizadas pela extração da substância de $10 \mathrm{mg}$ do pó das raizes, em $10 \mathrm{ml}$ de metanol, sofrendo agitação em banho de ultrasom, durante cinco minutos, para obtenção do extrato. Estes extratos foram analisados em Cromatógrafo Liquido de Alta Eficiência, utilizando coluna PEGASIL-ODS a temperatura ambiente e detector UV-VIS, com comprimento de onda igual a $280 \mathrm{~nm}$ e fase móvel metanol/água (7:3), com vazão de $1 \mathrm{ml} / \mathrm{minuto}$. Para a realização das análises comparativas, 
as porcentagens referentes aos teores de rotenona detectadas para o pó das raizes dos timbós, foram transformadas em arco-seno $\mathrm{v} x+1,0$.

Os individuos de $M$. domestica empregados foram capturados em duas granjas de galinhas poedeiras, uma localizada em Jaboticabal (SP) e outra em Brodowski (SP). De cada linhagem, 180 fêmeas foram isoladas e mantidas no laboratório para o fornecimento de ovos. Como Costa (1996), mostrou não haver efeitos diferenciais dos clones na emergência dos imagos das linhagens de moscas, estas foram consideradas conjuntamente, como repetições nos ensaios.

Os recipientes empregados para o desenvolvimento dos agrupamentos larvais foram de plástico transparente, com capacidade para $500 \mathrm{ml}$. Na tampa do recipiente foi confeccionada uma abertura de $3 \times 3 \mathrm{~cm}$ e no local foi fixado tecido de organza, para permitir a aeração e evitar a saida das larvas e imagos.

Os agrupamentos experimentais foram formados com 300 larvas com dois dias de idade, descendentes das moscas das populações naturais, cujos individuos foram colocados nos recipientes de plástico transparente, com meio de cultura $(31,4 \mathrm{~g}$ de farelo de trigo, 2,4 $\mathrm{g}$ de fermento, 1,5 $\mathrm{g}$ de leite em pó integral e $60 \mathrm{ml}$ de água destilada). Em cada agrupamento adicionou-se $0,7 \mathrm{~g}$ do pó da raiz do clone de timbó a ser testado, nas testemunhas não foi adicionado este produto. Foram realizadas cinco repetições para cada linhagem de mosca e para cada clone de tímbó, proporcionado 39 tratamentos (clones) com dez repetições, totalizando em todo o experimento 390 agrupamentos ou culturas experimentais.

\section{RESULTADOS}

Nos agrupamentos controle, as moscas de Jaboticabal e de Brodowski apresentaram médias e erros padrão para os números de imagos emergidos das culturas de $214,4 \pm 32,7$ e 227,0 $\pm 34,8$, respectivamente. A Tabela 1 mostra a identificação dos clones (números) de cada espécie de timbó, os locais de coletas dentro de cada região, com os respectivos teores de rotenona e os números médios de moscas produzidas nas culturas tratadas com o pó de suas raizes.

Uma análise comparativa entre os dados obtidos mostra que $D$. urucu apresentou uma média geral de rotenona para os clones de $3,76 \%$, a diferença entre o clone com maior conteúdo da substância (clone 862, região $\mathrm{F}$ ), com o de menor conteúdo (clone 41 , região $\mathrm{A}_{1}$ ) é de 388 vezes. Derris nicou apresentou média geral de $4,25 \%$, a diferença entre o clone com maior conteúdo da substância (clone 850, região $\mathrm{F}$ ), com o de menor conteúdo (clone 1044, região B) é de 112 vezes. A média obtida para Derris sp., foi de $0,18 \%$ e o clone 1048 da região $\mathrm{B}$, tem 145 vezes mais rotenona que o clone 783 da região $\mathrm{E}$.

Diferenças nos teores de rotenona apresentada pelas três espécies de timbó, mostraram valor significativo $(\mathrm{F}=4,95 ; \mathrm{P}<0,05)$. Derris 
Tabela 1. Relação dos clones de timbó (números), regiôes onde foram coletados, teores de rotenona nas respectivas raizes e produção média de moscas emergidas nos agrupamentos tratados com o pó das raizes e das testemunhas.

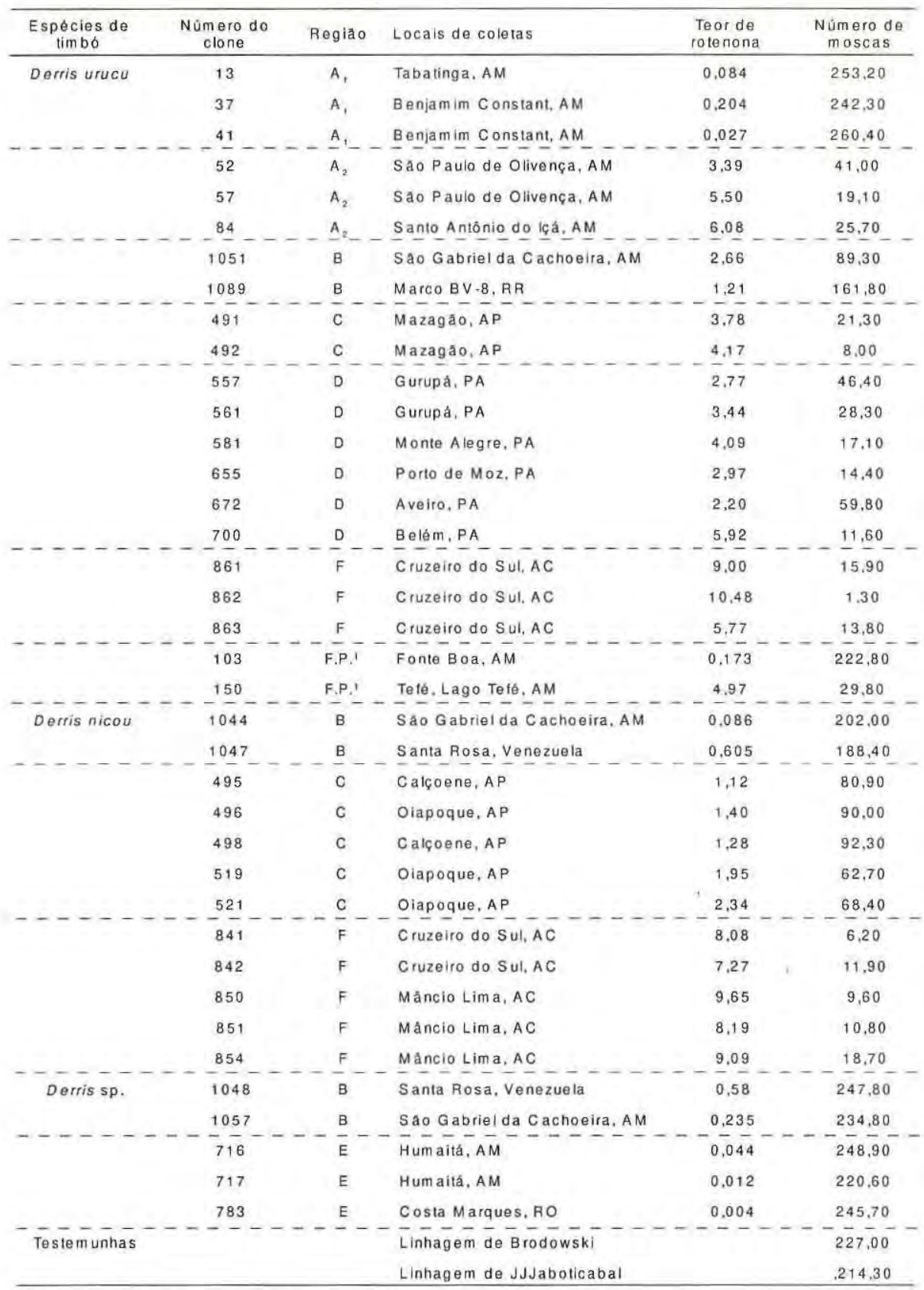

I= "Fundo do Prato" de acordo com Vanzolini (1970) 
urucu e $D$. nicou não diferiram e mostraram possuir maiores teores de rotenona que Derris sp. (Tab. 2).

As relações entre os efeitos dos teores de rotenona dos clones e os números de moscas emergidas dos agrupamentos, envolvendo as espécies D. nicou e D. иrucu (Figs. 2 e 3), indicam que os aumentos nos teores de rotenona nos clones de timbó correspondem a uma diminuição progressiva nos números de imagos emergidos.

A confirmação destas relações foi realizada através dos coeficientes de correlação " $r$ ". Os resultados mostraram correlações negativas significativas

$(\mathrm{P}<0,001)$, com $\mathrm{r}=-0,89$ para D. nicou e $\mathrm{r}=-0,81$ para $D$. urucu. $\mathrm{O}$ valor para Derris sp., $\mathrm{r}=0,30$ foi não significativo $(\mathrm{P}>0,05)$.

Para comparações, os valores de " $\mathrm{r}$ " foram transformados de acordo com Steel \& Torrie (1981) em z(r). Os resultados mostram que os dados obtidos para D. nicou e D. urucu são homogêneos. Diferença significativa foi obtida entre D. nicou e Derris sp. Quanto à $D$. urucu e Derris sp., o valor do teste " $t$ " não foi significativo (Tab. 3). Esta homogeneidade corresponde à distância de 0,03 para o nível de significância de $1,96(\mathrm{P}=0,05)$.

A deteç̧ão de eventuais variações entre as plantas de timbó da mesma espécie, provenientes das diferentes regiões, em relação aos teores de rotenona no pó de suas raizes, mostraram para Derris nicou $(\mathrm{F}=161,97 ; \mathrm{P}<0,001)$ е $D$. игиси $(\mathrm{F}=$ $16,44 ; \mathrm{P}<0,001)$, resultados altamente significativos, enquanto que para Derris sp., o resultado foi não significativo $(\mathrm{F}=10,08 ; \mathrm{P}>0,05)$, porém, muito próximo ao nível de significância $(\mathrm{F}=$ $10,13 ; \mathrm{P}=0,05)$. Os dados indicaram que os teores de rotenona encontrados em $D$. nicou diferiram significativamente entre as plantas provenientes das regiões $\mathrm{F}, \mathrm{C}$ e $\mathrm{B}$, indicando, respectivamente, que nas regiões $\mathrm{F}$ e $\mathrm{B}$ foram obtidos exemplares que apresentaram maior e menor teores para a substância (Tab. 4).

Em D. urucu, as variações foram mais complexas: das 15 diferenças possiveis entre as médias, seis foram significativas. Como na espécie anterior, os maiores teores de rotenona foram obtidos em plantas provenientes da região $\mathrm{F}$, as quais diferiram significativamente dos valores obtidos para os clones das regiões $\mathrm{A}_{1}, \mathrm{~B}$ e D; enquanto que as plantas provenientes da região $\mathrm{A}_{1}$ apresentaram os menores valores para o conteúdo de rotenona. Estes valores diferiram significativamente dos teores apresentados pelas plantas provenientes das regiões $\mathrm{A}_{2}, \mathrm{C}$ e $\mathrm{D}$.

\section{DISCUSSÃO}

Os teores de rotenona e os seus efeitos no controle das larvas de $M$. domestica foram importantes para a diferenciação dos clones das três espécies de timbó estudadas. Enquanto os dados mostraram uma homogeneidade média indicada pelas plantas de $D$. nicou e D. urucu, os valores apresentados por Derris sp. foram menores, em relação aos teores desta substância.

A importância de $D$. nicou foi enfatizada por Caminha Filho (1940), 
Tabela 2. Valores médios em porcentagens dos teores de rotenona nas espécies de timbó de origem sul-americana.

\begin{tabular}{lccc}
\hline Espécies & Valores em porcentagens & & DMS $^{2}$ \\
\hline Derris nicou & 4,26 & b & $\triangle_{21 \cdot 12}=3,55$ \\
Derris urucu & 3,76 & b & $\Delta_{21 \cdot 5}=4,88$ \\
Derris sp. & 0,18 & a & $\triangle_{12 \cdot 5}=5,22$ \\
\hline
\end{tabular}

1 = Médias seguidas pelas mesmas letras năo diferem significativamente, pelo teste de Tukey.

2 = diferença minima significativa.

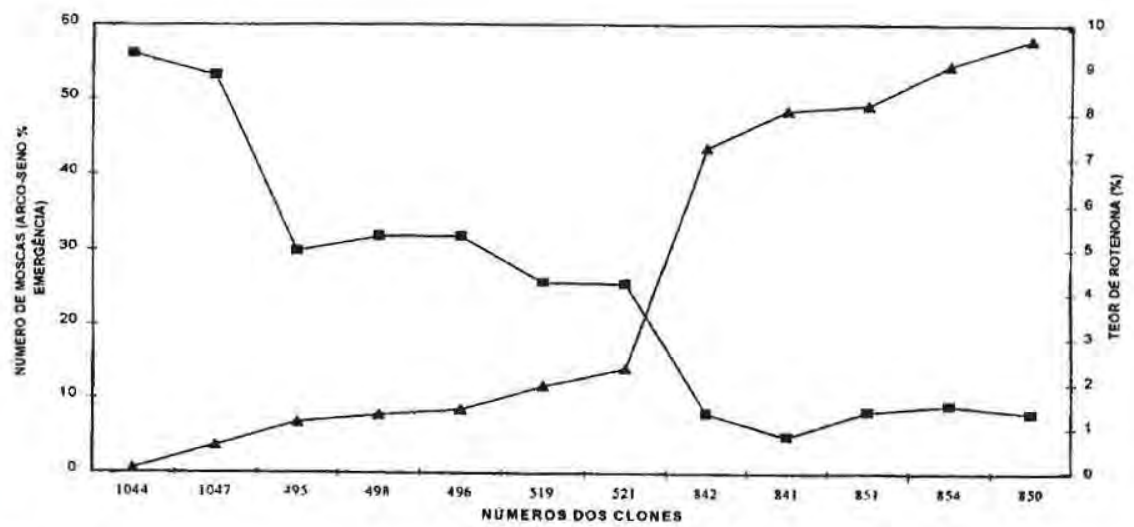

Figura 2. Relações entre os teores de rotenona dos clones (- - ) de Derris nicou e número de moscas (- $\longrightarrow$ ) emergidas dos agrupamentos.

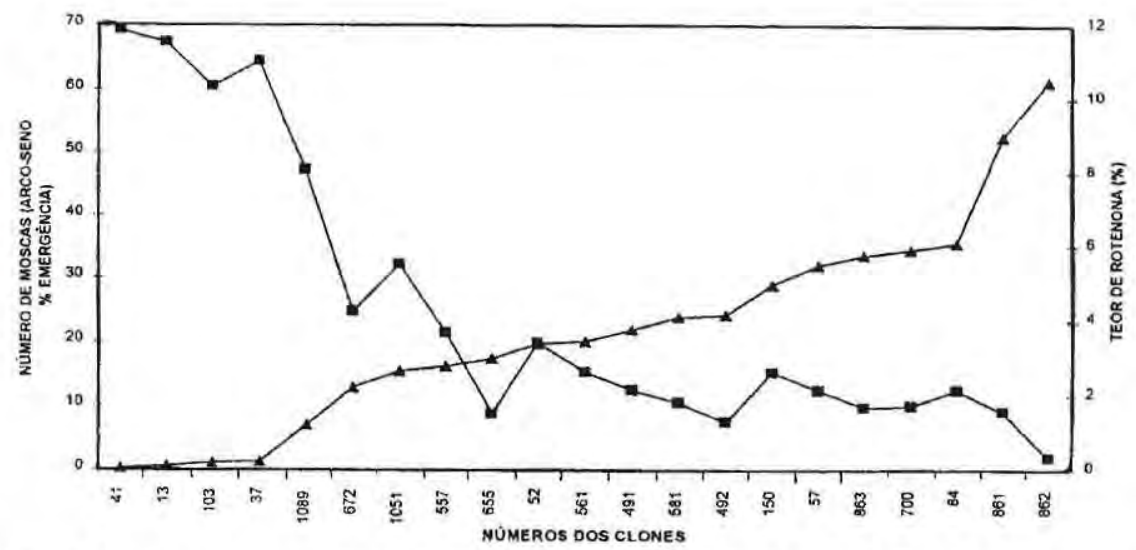

Figura 3. Relações entre os teores de rotenona dos clones $(\longrightarrow$ ) de Derris

игиси e número de moscas (—-) emergidas dos agrupamentos. 
Tabela 3. Resultados do teste aplicado para detectar diferenças entre os valores dos coefícientes de correlação (r) obtidos para as espécies sul-americanas de timbó.

\begin{tabular}{ccc}
\hline Comparaçöes entre os valores de " $\mathrm{r}$ " & Valores de " $\mathrm{t}$ " & $\mathrm{P}$ \\
\hline D. nicou $\times$ D. urucu & 0,72 & $>0,05$ \\
D. nicou $\times$ Derris sp. & 2,21 & $<0,05$ \\
D. urucu $\times$ Derris sp. & 1,93 & $>0,05$ \\
\hline
\end{tabular}

Tabela 4. Valores médios em porcentagens dos teores de rotenona para as plantas de timbó de cada região.

\begin{tabular}{|c|c|c|c|c|c|c|}
\hline \multirow{2}{*}{$\begin{array}{l}\text { Espécies } \\
\text { Derris nicou }\end{array}$} & \multirow{2}{*}{$\frac{\text { Regiões }}{\text { F }}$} & \multicolumn{4}{|c|}{ Valores em porcentagens ${ }^{1}$} & \multirow{2}{*}{$\frac{\mathrm{DMS}^{3}}{\Delta_{5 \cdot 5}=1,60}$} \\
\hline & & $8,46(5)^{2}$ & & & c & \\
\hline & C & $1,65(5)$ & & b & & $\triangle_{5 \cdot 2}=2,12$ \\
\hline & B & $0,34(2)$ & a & & & \\
\hline \multirow[t]{6}{*}{ Derris urucu } & $\mathrm{F}$ & $8,41(3)$ & & & c & $\Delta_{33}=3,79$ \\
\hline & $A_{2}$ & $4,99(3)$ & & b & C & $\Delta_{3 \cdot 6}=3,28$ \\
\hline & C & $3,97(2)$ & & $b$ & c & $\Delta_{2 \cdot 3}=4,23$ \\
\hline & D & $3,56(6)$ & & $b$ & & \\
\hline & B & $1,93(2)$ & a & $b$ & & \\
\hline & $A_{1}$ & $0,10(3)$ & a & & & \\
\hline
\end{tabular}

$1=$ Médias seguidas pelas mesmas letras năo diferem, significativamente, pelo teste de Tukey 2 = número de clones (entre parênteses) dentro de cada regiâo.

3 = diferença mínima significativa.

que apresentou maior quantidade de rotenona ( 15 a $17 \%)$, em relação a $D$. игиси (5 a 12\%), e o descreveu como sendo o mais ativo dos timbós (referiu-se a eles como pertencentes ao gênero Lonchocarpus). É possível que possam existir clones com essas proporções de principio ativo; mas, infelizmente eles não foram incluídos neste trabalho.

A classificação dos clones pelos teores de rotenona apresentados e as suas relações com o número de moscas emergidas dos agrupamentos experimentais enfatiza a importância da substância no controle das larvas. Os aumentos em rotenona nos clones de timbó, produziram reduções no número de imagos emergidos nas culturas.

Segundo Costa (1996), as plantas de $D$. nicou provenientes da mesma região, não apresentaram diferenças significativas em suas capacidades controladoras das larvas. Diferenças foram anotadas entre as plantas de regiões diferentes. Os clones provenientes da região $\mathrm{F}$ predominaram, em relação aos clones da região $\mathrm{C}$, e estes foram mais 
eficientes que aqueles da região B. As estimativas dos teores de rotenona destas plantas confirmaram que aquelas da região $\mathrm{F}$ apresentaram quatro vezes mais rotenona que os clones das regiões $\mathrm{C}$ e $\mathrm{B}$.

As plantas de D. urucu das regiões $A_{1}$ e $A_{2}$ apresentaram padrões diferenciais em relação aos teores de rotenona e nos seus efeitos na emergência de imagos dos agrupamentos experimentais. Estes grupos estão localizados em uma posição difícil, pois estão relativamente distanciados da região base, denominada de "refúgio do Napo", por Haffer (1969). Como descreveu este autor, na Amazônia, muitos "refúgios" poderiam ter existido ao longo dos rios mais volumosos, que não foram identificados em suas observações.

Devido ao conteúdo de rotenona e dos seus efeitos no controle das larvas, as plantas de $D$. urucu mostraram maior dificuldade na caracterização dos "refúgios florestais" propostos por Haffer (1967 e 1969), devido à sobreposição de valores encontrados para os clones das regiões $\mathrm{D}, \mathrm{C} \mathrm{eA}$. Entretanto, as regiões $\mathrm{F}, \mathrm{B}$ e $\mathrm{A}_{1}$, puderam ser muito bem diferenciadas de outras.

As plantas de D. итиси procedentes da região $\mathrm{F}$ (refúgio do Leste -Peruano) apresentaram como D. nicou, os maiores teores de rotenona e foram entre as amostras coletadas, as que mostraram maior capacidade para o controle das larvas de $M$. domestica.

Deste modo, a análise do conteúdo de rotenona em plantas de $D$. nicou e D. иrиси e os seus efeitos na emergência de imagos de $M$. domestica, mostraram diferenças entre plantas de regiões diferentes, dentro dessas espécies. Isso pode mostrar uma resposta favorável à teoria do isolamento que deve ter ocorrido em época anterior a atual na Amazônia. Fato descrito por Haffer (1969), Vanzolini (1970), Vuilleumier (1971) e Simpson \& Haffer (1978), com outras espécies. É evidente que diferenças entre os clones, especialmente os de $D$. urucu, no teor de rotenona e nos seus efeitos nas larvas, possam ter ligeiramente obscurecido a caracterização de certos "refúgios". Isso, entretanto, parece dever-se mais a localização inadequada do local de origem de algumas plantas, em relação aos locais originais dos "refúgios florestais" descritos por Haffer (1967 e 1969) e/ou pela longa distância geográfica entre os clones dentro de certas regiões. Por outro lado, alguns organismos podem ter uma distribuição mais abrangente que outros (Brown Júnior 1977, mostrou a existência de 38 "refúgios" para as borboletas do grupo Heliniini).

A denominação "fundo do prato"l foi usada por Vanzolini (1970) ao referirse às estruturas geográfica e física da Amazônia, pois a parte central é baixa, com padrões indefinidos de distribuições de organismos; enquanto os "refúgios florestais" são geralmente orográficos e periféricos. Infelizmente, só duas amostras de plantas provenientes dessa região foram empregadas, mas confirmaram positivamente, as afirmações do autor acima.

A especificidade observada nos 
testes para detecção das doses letais realizadas com clones de $D$. urucu e D. nicou (Costa et al. 1997), mais a seleção dos clones que melhor controlam as larvas dos agrupamentos experimentais, mostraram a importância do valor seletivo realizado nestas plantas, o que foi baseado na capacidade de controle destes insetos. Mais detalhes a respeito dos efeitos em $M$. domestica, regiões de origem e isolamento das plantas de timbó, serão publicados por Costa \& Bélo (1999).

As plantas de timbó apresentam grande capacidade de serem empregadas industrialmente, pois há possibilidade da extração, estabilização e padronização da rotenona, que é a principal substância contida em suas raizes. Segundo Lima (1987), as mesmas apresentam além da rotenona, os rotenóides (dequelina, tefrosina e toxicarol) que necessitam de mais pesquisas para se ter melhor conhecimento dos seus efeitos e que poderão servir de matéria-prima para a produção de inseticidas biológicos.

\section{Bibliografia citada}

Brown Junior, K.S. 1977. Centro de evolução, refúgios quaternários e conservação de patrimônios genéticos na região neotropical: padrões de diferenciação em Ithomiinae (Lepidoptera:Nymphalidae). Acta Amazônica, 7(1); 75-137.

Caminha Filho, A. 1940. Timbó e rotenona: uma riqueza nacional inexplorada. 2 ed.

Rio de Janeiro: Serviço de Informação Agrícola, 14 p.

Corbett, C.E. 1940. Plantas ictiotóxicas: farmacologia da rotenona. São Paulo: Faculdade de Medicina da Universidade de São Paulo, 157p. (mimeogr.).

Costa, J.P.C.da, 1996. Efeito da variabilidade de timbós de diferentes regiões da Amazônia em Musca dometica L. (Diptera: Muscidae). Dissertação de Mestrado, Faculdade de Ciências Agrárias e Veterinárias, Universidade Estadual Paulista, Jaboticabal (SP). $119 \mathrm{p}$.

Costa, J.P.C.da.; Bélo, M.; Barbosa, J.C. 1997. Efeitos de espécies de timbós (Derris spp.: Fabaceae) em populações de Musca domestica L. An. Soc. Entomol. Brasil., 26(1): 163-168.

Costa, J.P.C.; Bélo, M. 1999. Diferença entre as espécies de timbó ( Derris spp., Fabaceae) de diferentes regiões da Amazônia no controle da Musca domestica L. (submetido a apreciação).

Haffer, J. 1967. Speciation in Colombian forest birds west of the Andes. Am. Mus. Novit., 2294: 1-57.

Haffer, J. 1969. Speciation in Amazonian forest birds. Science, 165: 131-137.

Lima, R.R. 1987. Informações sobre duas espécies de timbó: Derris urucu (Killip et Smith) Macbride e Derris nicou (Killip et Smith) Macbride, como plantas inseticidas. Belém: EmbrapaCPATU (Documentos, 42), 23p.

Lima, R.R.; Costa, J.P.C.da, 1991. Registro de introdução de plantas de cultura précolombiana na Amazônia brasileira. B e l é m : E M B R A P A - C PAT U (Documentos, 58). 210p.

Link, R. P. 1965. Inseticides, In: Jones, L.M. (ed.). Veterinary pharmacology and therapeuutics. $3^{\text {a }}$ ed. Ames: Iowa State University. p. 702-726.

Pires, J.M. 1978. Plantas ictiotóxicas: aspecto da botânica sistemática. Anais do Simpósio de Plantas Medicinais do Brasil, 5, Campinas, ..., p.37-41,

Steel, R.G.D.; Torrie, J.H. 1981. Principles and procedures of statistics. McGraw-Hill International Book Company. $2^{\mathrm{a}}$ ed. $633 \mathrm{p}$. 
Simpson, B.B.; Haffer, J. 1978. Speciation patterns in the amazonian forest biota. Ann. Rev. Syst., 9: 497-518.

Vanzolini, P.E. 1970. Zoologia sistemática, geografia e a origem das espécies. São Paulo (1G - Série Teses e Monografias, 3). Instituto de Geografia, Universidade de São Paulo. 56p.

Vianna, C.H.M.; Odebrech, S.; Campello, A.P. 1979. Ação tóxica de extratos de Derris negrensis (timbó). Arq. Biol. Tecnol., 22: 95-110.

Vuilleumier, B.S. 1971. Pleistocene changes in the fauna and flora of south American. Science, 173: 771-780. 